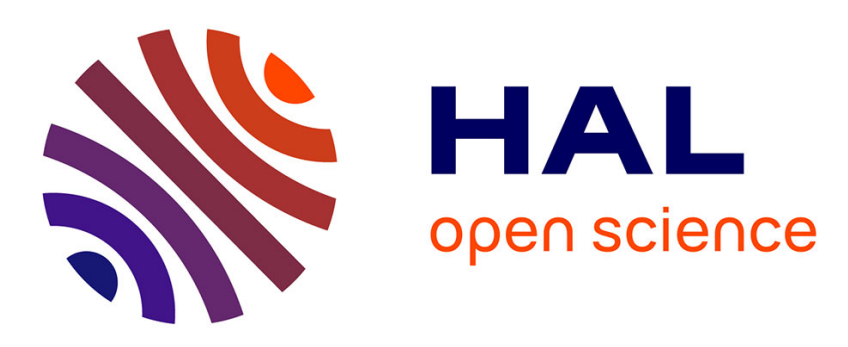

\title{
Testicular biodistribution of silica-gold nanoparticles after intramuscular injection in mice
}

Lara Leclerc, Jean-Philippe Klein, Valérie Forest, Delphine Boudard, Matteo

Martini, Jérémie Pourchez, Marie-Geneviève Blanchin, Michèle Cottier

\section{- To cite this version:}

Lara Leclerc, Jean-Philippe Klein, Valérie Forest, Delphine Boudard, Matteo Martini, et al.. Testicular biodistribution of silica-gold nanoparticles after intramuscular injection in mice. Biomedical Microdevices, 2015, 17 (4), pp.9968. 10.1007/s10544-015-9968-3 . hal-01216399

\section{HAL Id: hal-01216399 \\ https://hal.science/hal-01216399}

Submitted on 16 Oct 2015

HAL is a multi-disciplinary open access archive for the deposit and dissemination of scientific research documents, whether they are published or not. The documents may come from teaching and research institutions in France or abroad, or from public or private research centers.
L'archive ouverte pluridisciplinaire HAL, est destinée au dépôt et à la diffusion de documents scientifiques de niveau recherche, publiés ou non, émanant des établissements d'enseignement et de recherche français ou étrangers, des laboratoires publics ou privés. 


\section{Testicular Biodistribution of Silica-Gold Nanoparticles after}

\section{Intramuscular Injection in Mice}

Lara Leclerc ${ }^{1,2, *}$, Jean-Philippe Klein ${ }^{1,3, *}$, Valérie Forest $^{2}$, Delphine Boudard ${ }^{1,3}$, Matteo Martini ${ }^{4}$, Jérémie Pourchez ${ }^{2}$, Marie-Geneviève Blanchin ${ }^{4}$ and Michèle Cottier ${ }^{1,3}$

Affiliations:

${ }^{1}$ University of Lyon, F-42023 Saint-Etienne, Jean Monnet University, EA 4624 - LINA, SFR FED 4166 - IFRESIS

${ }^{2}$ Ecole Nationale Supérieure des Mines, CIS-EMSE, EA 4624 - LINA, SFR FED 4166 IFRESIS, F-42023 Saint-Etienne, France

${ }^{3}$ University Hospital CHU de Saint-Etienne, Histology-Embryology Department, France

${ }^{4}$ Institut lumière matière, UMR5306 Claude Bernard Lyon1 University -CNRS, University of Lyon 69622 Villeurbanne cedex, France

* These two authors contributed equally to this work

Suggested running head: Testicular biodistribution of nanoparticles in mice

Corresponding author:

Lara LECLERC

Laboratoire Interdisciplinaire d'étude des Nanoparticules Aérosolisées (LINA - EA 4624)

Faculté de Médecine Jacques Lisfranc, Université Jean Monnet

15 rue Ambroise Paré, 42023 Saint-Etienne cedex, France

Telephone / Fax number: + 33477421443 / +334774214 94, mail: lara.leclerc@univ-st$\underline{\text { etienne.fr }}$ 


\section{ABSTRACT \\ Background}

With the continuing development of nanomaterials, the assessment of their potential impact on human health, and especially human reproductive toxicity, is a major issue. The testicular biodistribution of nanoparticles remains poorly studied. This study investigated whether goldsilica nanoparticles could be detected in mouse testes after intramuscular injection, with a particular focus on their ability to cross the blood-testis barrier. To that purpose, wellcharacterized 70-nm gold core-silica shell nanoparticles were used to ensure sensitive detection using high-resolution techniques.

\section{Results}

Testes were collected at different time points corresponding to spermatogenesis stages in mice. Transmission electron microscopy and confocal microscopy were used for nanoparticle detection, and nanoparticle quantification was performed by atomic emission spectroscopy. All these techniques showed that no particles were able to reach the testes. Results accorded with the normal histological appearance of testes even at 45 days post sacrifice.

\section{Conclusion}

High-resolution techniques did not detect 70 -nm silica-gold nanoparticles in mouse testes after intramuscular injection. These results are reassuring about the safety of nanoparticles with regard to male human reproduction, especially in the context of nanomedicine.

\section{KEY WORDS}

Nanoparticles, mice, testes, intramuscular injection, reproductive toxicity. 


\section{INTRODUCTION}

During the last 20 years, nanotechnology has drastically evolved, leading to increasing population-level exposure to engineered nanoparticles and nanomaterials. A wide variety of fields, such as the textile industry, computer sciences, cosmetics, and medicine, have taken advantage of nanostructured compounds. Their intrinsic small size and high surface reactivity (Oberdörster et al. 1994), which could allow specific interactions with biological structures, raise concerns in the scientific community about potential environmental or human health toxicity (Peralta-Videa et al. 2011). Concomitantly, declining sperm counts have been documented in most Western countries (Lackner et al. 2005; Rolland et al. 2013). Thus, concerns have been raised about the ability of nanoparticles to impair human reproduction, especially male fertility (Ema et al. 2010; Lan and Yang 2012). Two types of studies have focused on this topic: (1) testicular biodistribution and biopersistence studies and (2) testicular biotoxicity studies.

With regard to biodistribution, all studies performed in mice or rats have concluded that very limited amounts of nanoparticles can reach the testes regardless of the exposure routes (inhalation, intravenous, oral) and the physico-chemical properties of nanoparticles, including carbon nanotubes and gold, cerium oxide, silica and silver nanoparticles measuring between 10 and $5000 \mathrm{~nm}$ (Bai et al. 2010; Balasubramanian et al. 2010; Geraets et al. 2012; De Jong et al. 2008; Lankveld et al. 2010; Lan and Yang 2012; Morishita et al. 2012; Park et al. 2010; van der Zande et al. 2012). Most studies agree that the testes is a minor target for distribution of nanoparticles compared with other organs (Geraets et al. 2012; De Jong et al. 2008; Lankveld et al. 2010). However, the studies are not entirely consistent. Nanoparticle size variation may explain some of these discrepancies (De Jong et al. 2008; Morishita et al. 2012; Park et al. 2010), and exposure route and particles agglomeration may also contribute to the differences. Testicular biopersistence of nanoparticles seems to depend on exposure route; for 
instance, biopersistence of silver nanoparticles was less than 2 weeks following intravenous exposure (Lankveld et al. 2010), but more than 4 months after oral exposure (Lee et al. 2013; van der Zande et al. 2012). Furthermore, some authors have suggested that nanoparticles could cross the blood-testis barrier, but only Moroshita et al. (2012) have provided evidence. Their study in mice demonstrated the presence of nanoparticles in spermatocytes after an intravenous injection of 70-nm silica nanoparticles.

Regarding testicular biotoxicity, three concerns have been raised: reduction in daily sperm production (Gromadzka-Ostrowska et al. 2012; Xu et al. 2014; Yoshida et al. 2009), although this outcome is not reported by all authors (Bai et al. 2010); increase in testosterone synthesis (Li et al. 2009; Ramdhan et al. 2009; Tassinari et al. 2014; Yoshida et al. 2009); and the appearance of histological abnormalities. Histological analysis of mice testes after multiple intravenous injections of carbon nanotubes showed damage to seminiferous tubules, particularly reductions in the thickness of the germinative layer and the number of germ stem cells; however, the abnormalities resolved after 60 days (Bai et al. 2010). GromadzkaOstrowska et al. (2012) showed that intravenous injection of 200-nm silver nanoparticles in rats induced enlargement of intercellular spaces and vacuolization of the germinal epithelium, while 20-nm silver nanoparticles did not have the same effects. In another study, inhalation of carbon black nanoparticles of $14-95 \mathrm{~nm}$ in mice led to the degeneration of some seminiferous tubules compared to the control group (Yoshida et al. 2009).

In contrast, Morishita et al. (2012) revealed that although intravenously injected $70 \mathrm{~nm}$ silica particles reached the testes, they did not trigger any histological abnormalities in mice. Similarly, Chen et al. (2013) found no testicular histological abnormalities after intraperitoneal injection of pegylated gold nanoparticles in mice, (Chen et al. 2013) and Xu et al. (2014) did not find any histological changes in mice testes after 13 days of intravenous 
exposure to silica nanoparticles, apart from reversible ultrastructural changes in mitochondria. Finally, no study has found any impact of nanoparticles on testis weight.

In a previous study, we demonstrated the presence of individual 450-nm fluorescent particles in the testes after intramuscular injection in mice (Klein et al. 2013). The intramuscular route was specifically chosen because it is normally used for vaccine delivery, and nanoparticles are increasingly being used and explored as vaccine adjuvants (Gregory et al. 2013; Oyewumi et al. 2010; Peek et al. 2008). However, to our knowledge, no other reports have been published about testicular biodistribution or biotoxicity of nanoparticles used as vaccine adjuvants. Given that some works have shown that smaller nanoparticles induce better immunogenicity (Fifis et al. 2004) and trigger better cellular immune responses (Oyewumi et al. 2010), we studied the testicular biodistribution of smaller particles (70-nm gold core-silica shell nanoparticles) after intramuscular injection in mice. The hybrid nature of these particles allowed (i) an easy detection by high-resolution transmission electron microscopy (TEM) and inductively coupled plasma-atomic emission spectroscopy (ICP-AES) due to the gold core, and (ii) fluorescence detection due to the FITC dyes encased within the silica shell. The histological morphology of testes was also examined to identify any possible morphological alterations in the blood-testis barrier by the presence of particles; the cellular integrity of the seminiferous epithelium was also verified. 


\section{METHOD}

\section{Nanoparticles synthesis and characterization}

All colloidal solutions used for biodistribution assays were provided by the ILM laboratory and consisted of 5-nm gold cores coated with a 65-nm polysiloxane shell ( $\mathrm{SiOx}$ ) doped with fluorescein molecules. The particle surface was functionalized by the addition of -COOH groups to ensure long-term stability for biological assays. Fluorescein dyes covalently entrapped within a silica matrix were chosen for their strong emission luminescence at 518 $\mathrm{nm}$ as tracers for epifluorescence microscopy detection (Olympus). Briefly, the colloidal solution was prepared according to a previous method (Martini et al. 2009) based on sol-gel synthesis in a water/oil microemulsion to control the size, dye amount, and homogeneity of the particle population. Thereafter, particles were purified by ultrafiltration using $300 \mathrm{kDa}$ PES membranes (with a purification rate higher than $10^{8}$ ) and then dispersed in aqueous solution $\left(20\right.$ g.L $\left.{ }^{-1}\right)$ for further dilutions.

The detailed structural and morphological examination of samples was carried out by transmission electron microscopy (TEM) using a HITACHI H-800 microscope. The samples were prepared by depositing a drop of diluted colloidal solution onto a carbon grid (200 mesh) and allowing the solvent to evaporate at room temperature. Measurements of the hydrodynamic size and the Zeta-potential were performed on a Dynamic Light Scattering apparatus (Zetasizer NanoZS, Malvern).

\section{Animals}

Eighteen male C57BL6 mice (Centre d'Elevage René Janvier, France) were housed at the animal experimental platform of the Jean Monnet University (PLEXAN - Plateforme d'EXpérimentation et ANalyses, Agreement number 42-18-0801) and all experiments were performed in compliance with the French laws and institutional guidelines. An authorization 
approving the experiments was obtained from the French Ministère de l'Enseignement Supérieur et de la Recherche (MESR, field number 00462.02). Experiments were started when the mice were between 6 and 8 weeks of age.

\section{Intramuscular injection of nanoparticles}

Particles were diluted in physiological water and a volume of $50 \mu \mathrm{L}$ was injected in each tibialis anterior muscle of 15 mice, for a total amount of $1.6 \times 10^{13}$ particles per mouse. Three mice received an injection containing only physiological water and constituted the negative control group.

\section{Biological samples}

The 15 injected mice were divided into five groups and sacrificed by cervical dislocation after $1 \mathrm{~h}$ (H1), 4 days (D4), 21 days (D21), 36 days (D36), or 45 days (D45) post injection. These time points coincide with the duration of spermatogenesis in mice. For each series, testes were excised and prepared for characterization (ICP-AES, histological analysis, TEM, and confocal microscopy); mice from the control group were sacrificed after 45 days. To that purpose testes were obtained and then subdivided in several pieces for the various investigations.

Mice were immobilized and their urine was collected in a tube; blood was collected by retroorbital puncture just before sacrifice. The silica-gold nanoparticles' structural stability as well as their capacity to translocate from the site of injection were assessed by TEM in whole blood and by ICP-AES in urine samples, respectively.

\section{Induced Coupled Plasma-Atomic Emission Spectroscopy}

At each time point, testes were excised, weighted, mechanically dilacerated, and incubated overnight in a bath of $6 \mathrm{M} \mathrm{HCl}$ under agitation. Samples were then dried at $90^{\circ} \mathrm{C}$ and diluted 
in $2 \mathrm{M} \mathrm{HCl}$ before ICP analyses of the amount of gold using adequate controls (ICP-AES, Horiba Jobin Yvon Activa-S coupled with Jobin Yvon AS 421 autosampler).

The same protocol was used to evaluate the levels of gold in urine samples.

\section{Histological analysis}

Freshly excised testes were cut and fixed in Japanese fixator (3\% paraformaldehyde, 2\% sodium cacodylate, and $0.2 \%$ picric acid), then rinsed three times ( 5 min per wash) in sodium cacodylate, and finally dehydrated in two acetone baths (30 min each). Samples were impregnated overnight at $4^{\circ} \mathrm{C}$ in glycolmethacrylate (GMA) and then embedded in resin (GMA and aniline/propanol solution) at $4^{\circ} \mathrm{C}$ until complete polymerization.

Nonadjacent 3- $\mu$ m-thick sections of resin-fixed testes were prepared using a microtome (Leica RM 2065). The sections were placed on slides (Superfrost Plus, Thermo Scientific), stained with Masson Trichrome stain, dried, and mounted with an appropriate mounting medium (Eukitt@: Sigma, France). Since the mouse testis is a homogenous organ, we considered each section as representative of the whole testis. Observations were performed with an optical microscope (Nikon Eclipse 80i) coupled with a digital-sight Nikon camera and a series of microscope objectives $(\times 10, \times 40, \times 60)$. The samples were randomly and blindly observed.

\section{Transmission Electron Microscopy}

Freshly excised testes were fixed with a solution of $2 \%$ paraformaldehyde, $4 \%$ glutaraldehyde, $0.2 \mathrm{M}$ monosodium dipotassium buffer at $\mathrm{pH}$ 7.4. Testes samples of about 1 $\mathrm{mm}^{3}$ were then cut and washed five times in $0.175 \mathrm{M}$ monosodium dipotassium buffer and postfixed in $2 \%$ osmium tetroxide. After washing, samples were dehydrated by graded series 
of ethanol baths $(30 \%, 50 \%, 70 \%, 90 \%, 100 \%)$ with a last bath in a transition solvent (propylene oxide) before embedding in EPON 812 resin. Samples were then sliced using an ultramicrotome (Leica, MTXL).

For conventional TEM studies, 65-nm-thick slices were deposited on Formvar MESH Grid and contrasted by means of uranyl acetate and lead citrate. TEM observations, up to magnification $\times 150,000$, were carried out on the samples using a H-800 electron microscope (Hitachi) at $75 \mathrm{kV}$. Once filtrated and deposited on copper grids, whole blood samples were also analyzed by TEM without the use of contrasting agents.

For elemental gold analysis, slices were deposited onto TEM copper grids covered with carbon film and contrasted only by means of uranyl acetate. High-resolution (HR) TEM studies with electron dispersive X-ray (EDX) analyses were performed using a TOPCON electron microscope capable of a 0.18-nm point to point resolution (EM-002B Ultra High Resolution Analytical Electron Microscope) operated at $120 \mathrm{kV}$ equipped with EDX detector (Thermo Electron Corporation Noran SI X system). For first step HR imaging some slices were deposited onto TEM copper grids covered with carbon film and contrasted only by means of uranyl acetate. For elemental Au analysis, slices deposited on carbon film grids were prepared without coloration: no uranyl acetate nor lead citrate.

\section{Confocal microscopy}

A portion of the samples was placed in a cryopreservative solution (Tissue-Tech®: Sakura, Netherlands) and cryoconserved in liquid nitrogen. For confocal analyses, $10-\mu \mathrm{m}$ slides were cut with a cryostat at $-20^{\circ} \mathrm{C}$ (Leica CM-3000). Sections were then dried, mounted with an appropriate mounting medium (Fluokeep ${ }^{\circledR}$ : Argene, France), and stored at $4^{\circ} \mathrm{C}$ in the dark. 
Analyses were performed using a confocal microscope TCS-SP2 (Leica ${ }^{\circledR}$ Microsystems, Heidelberg, Germany) coupled to an inverted microscope with oil immersion objective (HCX APO L U-V-I 639 1.2 NA), and fitted with acousto-optical beam splitter covering a 350-850 nm wavelength range. 


\section{RESULTS}

\section{Nanoparticles characterization}

Physico-chemical features of the nanoparticles are reported in Figure 1. The monoexponential correlation function obtained allows us to determine the Z-average, polydispersity index, and zeta potential values before the application. The size distribution of nanoparticles (Fig. 1A) assessed by dynamic light scattering showed an average diameter of $70 \mathrm{~nm}$ with negligible variation. The good homogeneity of the particle size as well as the absence of particle agglomeration allowed the use of colloidal solutions for biodistribution assays. As expected, the zeta potential at physiological $\mathrm{pH}$ (Fig. 1B) was negative and equal to $-13 \mathrm{mV}$, due to the covalently grafted carboxylate surface groups. On TEM images (Fig. 1C) the gold core was highlighted by dark-contrast spot at the center of the particle, this picture also illustrates the good reproducibility of the nanoparticle synthesis. The fluorescent signal of the particles was validated using epi-fluorescent microscopy (Fig. 1D).

The presence of silica-gold nanoparticles in whole blood samples was assessed by TEM and in urine samples by ICP-AES, as shown in Figure 2. A significant amount of gold was detected in urine at $\mathrm{H} 1$ and D4 (Fig. 2A) and nanoparticles were observed in blood samples at D4 and D21 (Fig. 2B), indicating that nanoparticles were able to leave the site of injection and maintain their integrity over time in biological media.

\section{ICP quantification}

The presence of gold was quantified in the testes using the ICP-AES technique. A dilution series of nanoparticles was performed to assess the detection limit of the technique $(1 / 10$, $1 / 100,1 / 500,1 / 1000,1 / 10,000,1 / 100,000)$. These preliminary data showed that under a $1 / 100,000$ dilution the ICP technique was not able to detect gold in the samples, defining a threshold of $1.6 \times 10^{8}$ nanoparticles (data not shown). 
Figure 3 summarizes the estimated amount of gold in the testes (expressed in $\mathrm{ppm}$ ) normalized by the organ weight. A positive control corresponding to the same amount of nanoparticles injected directly into a freshly excised testis was used. Regardless of the time of analysis after exposure, gold amounts were very weak, indicating a negligible testicular presence of the nanoparticles after intramuscular injection. A calibration curve revealed that less than 1 out of $10^{5}$ nanoparticles reached the testis.

\section{Histological observations}

After Masson Trichrome staining, tissue sections from control mice and mice injected with the nanoparticles were compared to investigate potential histological alterations (Fig 4). Regardless of the time of analysis, the testis generally appeared well preserved, without morphological alterations. Even after 45 days of exposure, no tubule necrosis or histological disorganization at the level of germinal cells and spermatozoids were observed, either in the intertubular spaces with blood vessels or in the Leydig cells. Moreover, the Sertoli cells, which form the blood-testis barrier that might be able to internalize nanoparticles, appeared morphologically normal and abundant. The proportion of each cell type seemed normal at each time of exposure (Fig $4 \mathrm{~A}$ and B).

Only small alterations of tubules were occasionally observed on the slides, irrespective of the time of analysis. Similar alterations were also seen on the control slides, indicating no correlation with the nanoparticle exposure (Fig 4 C, D, E). Some giant germinal cells with large vacuolized cytoplasm as well as desquamation of the germinal cells in the lumen of tubules were observed.

A particular alteration was noticed on one mouse at D4, with empty tubules showing only Sertoli cells without germinal cells (Fig 4F). Although this type of alteration is compatible 
with a toxic event, in this case it was considered to be an artifact because it was observed very soon after exposure and was observed only for a single mouse.

\section{TEM studies}

TEM observations can give information about the inner morphology of testes as well as the spatial distribution of nanoparticles present in the tissues. Analyses were performed on the testes with a multiscale approach to detect whether nanoparticles were present. The mouse testis is a homogenous organ, with repetitions of the morpho-functional unit corresponding to the seminiferous tubule surrounded by the intertubular spaces, which contain the Leydig cells. Thus, it was assumed that each sliced section was representative of the whole testis. To visualize all the structures at each time point, 40 slices per sample were observed. This reduced any systematic error due to the specific choice of a few slices.

First, conventional (Hitachi microscope) TEM observations (Fig. 5) were performed for the detection of nanoparticles in the samples. All slices were carefully imaged and entirely inspected. Cellular structures such as cytoplasmic vacuoles in Sertoli and germinal cells and the intertubular spaces containing the Leydig cells were studied to find nanoparticles. Particular care was taken in examining the seminiferous tubules (two to three tubules sections contained in each slice) to determine if nanoparticles can cross the blood-testis barrier. For each time point of the spermatogenesis process, no nanoparticles were identified in any of the regions of interest. One fourth of the sections were selected for HR imaging coupled with EDX analysis using the TOPCON microscope (Fig 6). No contrast metallization of the slices was performed to suppress the background noise from lead and uranyl compounds, and consequently the signal of the copper grid was detected on the spectra. Reference data were recorded from a control sample (Fig 6A) obtained from a section slice covered by a drop of the silica-gold nanoparticle suspension. An area imaged in Fig. 6A displays the characteristic 
features of the silica-gold nanoparticles, and the EDX spectrum recorded on the corresponding slice region exhibits a well-marked peak resulting from the contributions of the silicon and gold elements in addition to the peaks from copper and osmium. The results from the D45 samples reproduced in Figure 6B are representative of those for all other time points. Again the slices were carefully inspected by high resolution/high magnification imaging (Fig. 6B) and no indication of the nanoparticles (morphology, size, contrast) could be identified on the images. The corresponding EDX spectra showed only a weak peak, corresponding to the osmium post-fixing agent included in the samples during the preparation, but no silicon-gold peak. Similar spectra were observed for series of samples, revealing the absence of the silicon-gold signal. The problem of the Minimum Detectable Mass (MDM) of a given element in a given matrix has been investigated both theoretically and experimentally (Reimer 1989; Williams 1984). For the TEM / EDX system used here and operated in the conditions of the present studies the MDM of a heavy element like $\mathrm{Au}$ in a biological thin foil (light elements with a very small concentration of osmium) should be a few $10^{-19} \mathrm{~g}$, whereas the mass of one $5 \mathrm{~nm}$ gold core is more than $10^{-18} \mathrm{~g}$. Thus the cross correlation of HR imaging inspection with corresponding EDX analysis supports the conclusion that there is no gold core nanoparticle in the observed sections.

\section{Confocal observations}

To optimize fluorescein emission detection, reflection imaging was used to visualize testes, avoiding tissue autofluorescence as demonstrated in our previous study (Klein et al. 2013). For all samples, five slices were screened for each time point without being able to distinguish any fluorescent nanoparticles (Fig 7A-7F). Cellular structures appeared fluorescent on the samples, even on the negative control within the intertubular spaces. These areas corresponded to red blood cells, which are known to be autofluorescent. 


\section{DISCUSSION}

The aim of this study was to assess the testicular biodistribution of 70-nm gold-silica nanoparticles after intramuscular injection in mice and to define their histological reproductive toxicity. As previously mentioned, the use of nanoparticles as a vaccine adjuvant is promising due to their ability to enhance immune response. However, safety has been poorly documented, especially considering the intramuscular exposure route. This study was intended to address this issue. The nanoparticles were designed to be detectable by several complementary methodologies (epi-fluorescence and confocal microscopy, ICP-AES, electron microscopy). All of the physico-chemical properties as well as the morphology and stability of tracers were validated before the nanoparticles were injected into mice. The ability of nanoparticles to enter the circulatory system and their stability in the blood were assessed by observing them in blood samples at D4 and D21 using TEM. It was also shown that significant amounts of particles were excreted through urine at H1 and D4.

Here an original multiscale approach using high-resolution methodologies (confocal microscopy, ICP-AES, TEM, and EDX) was achieved. Regarding qualitative techniques, especially confocal microscopy, though testicular autofluorescence remained a problem despite the use of reflection imaging, the present image features do not seem indicative of the presence of nanoparticles. Testicular autofluorescence is mainly in the visible spectrum, so we are currently developing Cyanine 5.5-doped nanoparticles of the same size $(70 \mathrm{~nm})$ : their emission is centered in the near infrared region, which will help us to reduce background noise and obtain more reliable results using confocal microscopy. ICP-AES detection threshold for the nanoparticles used in this study was estimated to be $1.6 \times 10^{8}$ nanoparticles, which corresponded to $1 / 100,000$ of the injected dose. The use of other measurement techniques such as ICP-MS could improve the sensitivity of nanoparticles detection by reducing this threshold. Despite the limitations of confocal microscopy and ICP-AES 
techniques their correlation with the conclusive results of present imaging and analysis TEM studies supported the conclusion that nanoparticles were absent in the testes.

Previous biodistribution studies showed that small amounts of sub-micrometric particles reached the testes, depending on experimental conditions. For example, after a single intravenous injection of $5 \mathrm{mg} / \mathrm{kg}$ of carbon nanotubes in mice, $151 \mathrm{ng}$ of particles were found in testes $24 \mathrm{~h}$ later, corresponding to about $0.1 \%$ of the injected particles (Bai et al. 2010). Lankveld et al. (2010) found comparable results after intravenous injection of 20-nm silver nanoparticles for 5 days. Testicular distribution of $80-\mathrm{nm}$ and $110-\mathrm{nm}$ particles was also observed in very low amounts. De Jong et al. (2008) reported that after intravenous injection of $80 \mu \mathrm{g}$ of $10-\mathrm{nm}$ gold nanoparticles in rats, $55 \mathrm{ng} / \mathrm{g}$ was found in the testes, which means 1 out of 100,000 particles. Balasubramanian et al. (2010) observed similar results with a small amount of 20-nm gold nanoparticles ( $3 \mu \mathrm{g}$ per rats) injected intravenously. Lee et al. (2013) also showed that about one 10 - or 25 -nm silver nanoparticle out of $10^{6}$ was able to reach the rat testis after 4 weeks of oral exposure. Our previous results showed that 1 out of $10^{7} 450-\mathrm{nm}$ particles injected by the intramuscular route was found in the testes (Klein et al. 2013). In the present study, using smaller particles $(70 \mathrm{~nm})$, it was observed that less than 1 out of $10^{5}$ nanoparticles was able to reach the testes after intramuscular injection. This result seems in agreement with other quantitative studies about particles larger than $50 \mathrm{~nm}$ injected by intravenous route, which tend to be either not distributed in testes or distributed in very small amounts (Morishita et al. 2012). Similarly, De Jong et al. (2008) also demonstrated that 50-, 100-, and 250-nm particles were unable to reach testes(De Jong et al. 2008). Therefore, despite different exposure routes, the results tend to support the same conclusion, suggesting that nanoparticle size could be a key point for differences in testicular biodistribution. 
In this study, we also confirmed that no morphological alterations of the testes were apparent as a result of exposure to nanoparticles. This result is in accordance with Morishita et al. (2012) and Chen et al. (2013).

To put the low testicular distribution of nanoparticles observed in this study into perspective with the risk of reproductive toxicity of nanoparticles in real-life situations, in vitro studies are useful. Indeed, these investigations are complementary to in vivo models and provide additional information. The use of cell lines is particularly appropriate for the investigation of the mechanisms or thresholds of toxicity. Asare et al. (2012) showed that after murine testicular cells underwent a $48-\mathrm{h}$ exposure to $10 \mu \mathrm{g} / \mathrm{ml}$ of silver nanoparticles, the metabolic activity measured by MTT assay was reduced and the number of necrotic cells was slightly increased; however, other toxic effects, such as apoptosis and DNA damage, were only observed for higher concentrations (Asare et al. 2012). It was also shown that carbon black nanoparticles at a concentration of $10 \mu \mathrm{g} / \mathrm{ml}$ were able to increased StAR expression in Leydig cells, which could cause an increase in testosterone synthesis. Smaller concentrations of $\mathrm{TiO}_{2}$, carbon black, or diesel exhaust particles had no impact on Leydig cells (Komatsu et al. 2008), whereas Bradich-Stolle et al. (2005) showed that silver (Ag, $15 \mathrm{~nm}$ ), molybdenum $\left(\mathrm{MoO}_{3}, 30 \mathrm{~nm}\right)$, and aluminum $(\mathrm{Al}, 30 \mathrm{~nm})$ nanoparticles at a concentration of $5 \mu \mathrm{g} / \mathrm{ml}$ increased leakage of lactate dehydrogenase from germ-line stem cells (Braydich-Stolle et al. 2005). Taken together, these data suggest that studies using different kinds of nanoparticles, different cells types, and so forth yield varying results, making it difficult to draw definitive conclusions. However, a clear trend is emerging: in most of the published papers, toxic effects were observed only for high concentrations of particles (above $5 \mu \mathrm{g} / \mathrm{ml}$ ). Thus, considering that our results showed that less than 1 out of $10^{5}$ particle reached the testis after an intramuscular injection, this route can be considered as safe as long as the quantity of nanoparticles injected into mice does not exceed $50 \mathrm{mg}$. This value appears too high to be 
clinically relevant or even realistic. Therefore, we may extrapolate that the probability of toxicity on the reproductive system is very low.

\section{CONCLUSIONS}

Negatively charged 70-nm silica nanoparticles were injected intramuscular to study the biodistribution and effects of colloidal solution on testes. Different physico-chemical detection techniques confirmed the absence of nanoparticles in this region of interest. Moreover, no histological toxicity signs were observed. This study is reassuring regarding reproductive toxicity concerns associated with the possible use of nanoparticles in intramuscular injections, especially as vaccine adjuvants. Further in-depth analyses with different exposure routes and various nanoparticle chemistries (e.g., varying size, surface, composition) should be performed to confirm the reproductive safety of nanoparticles. 


\section{Acknowledgments}

The authors would like to acknowledge the financial support of the French Rhône Alpes Region through the ARC3 Environnement Research Program. They are grateful to Dr N. Blanchard for his help in operating the TOPCON microscope at ILM Institute.

\section{Authors' contributions}

LL, JPK, MGB and MC contributed to the conception and the design of research.

LL, JPK, VF, DB, MM and MGB materially participated in the research experiments analysis and interpretation of data.

LL and JPK were involved with drafting article.

All authors were involved with reviewing the article.

All authors have approved the final version of this article.

\section{Compliance with Ethical Standards}

All experiments were performed in compliance with the French laws and institutional guidelines. An authorization approving the experiments was obtained from the French Ministère de l'Enseignement Supérieur et de la Recherche (MESR, field number 00462.02). Conflict of Interest: The authors declare that they have no conflict of interest. 


\section{REFERENCES}

N. Asare, C. Instanes, W. J. Sandberg, M. Refsnes, P. Schwarze, M. Kruszewski, and G. Brunborg, Toxicology 291, 65 (2012).

Y. Bai, Y. Zhang, J. Zhang, Q. Mu, W. Zhang, E. R. Butch, S. E. Snyder, and B. Yan, Nat. Nanotechnol. 5, 683 (2010).

S. K. Balasubramanian, J. Jittiwat, J. Manikandan, C.-N. Ong, L. E. Yu, and W.-Y. Ong, Biomaterials 31, 2034 (2010).

L. Braydich-Stolle, S. Hussain, J. J. Schlager, and M.-C. Hofmann, Toxicol. Sci. Off. J. Soc. Toxicol. 88, 412 (2005).

J. Chen, H. Wang, W. Long, X. Shen, D. Wu, S.-S. Song, Y.-M. Sun, P.-X. Liu, S. Fan, F. Fan, and X.-D. Zhang, Int. J. Nanomedicine 8, 2409 (2013).

M. Ema, N. Kobayashi, M. Naya, S. Hanai, and J. Nakanishi, Reprod. Toxicol. Elmsford N 30, 343 (2010).

T. Fifis, A. Gamvrellis, B. Crimeen-Irwin, G. A. Pietersz, J. Li, P. L. Mottram, I. F. C. McKenzie, and M. Plebanski, J. Immunol. 173, 3148 (2004).

L. Geraets, A. G. Oomen, J. D. Schroeter, V. A. Coleman, and F. R. Cassee, Toxicol. Sci. Off. J. Soc. Toxicol. 127, 463 (2012).

A. E. Gregory, R. Titball, and D. Williamson, Front. Cell. Infect. Microbiol. 3, 13 (2013).

J. Gromadzka-Ostrowska, K. Dziendzikowska, A. Lankoff, M. Dobrzyńska, C. Instanes, G. Brunborg, A. Gajowik, J. Radzikowska, M. Wojewódzka, and M. Kruszewski, Toxicol. Lett. 214, 251 (2012).

W. H. De Jong, W. I. Hagens, P. Krystek, M. C. Burger, A. J. A. M. Sips, and R. E. Geertsma, Biomaterials 29, 1912 (2008).

J.-P. Klein, D. Boudard, J. Cadusseau, S. Palle, V. Forest, J. Pourchez, and M. Cottier, Biomed. Microdevices 15, 427 (2013).

T. Komatsu, M. Tabata, M. Kubo-Irie, T. Shimizu, K.-I. Suzuki, Y. Nihei, and K. Takeda, Toxicol. Vitro Int. J. Publ. Assoc. BIBRA 22, 1825 (2008).

J. Lackner, G. Schatzl, T. Waldhör, K. Resch, C. Kratzik, and M. Marberger, Fertil. Steril. 84, 1657 (2005).

D. P. K. Lankveld, A. G. Oomen, P. Krystek, A. Neigh, A. Troost-de Jong, C. W. Noorlander, J. C. H. Van Eijkeren, R. E. Geertsma, and W. H. De Jong, Biomaterials 31, 8350 (2010).

Z. Lan and W.-X. Yang, Nanomed. 7, 579 (2012).

J. H. Lee, Y. S. Kim, K. S. Song, H. R. Ryu, J. H. Sung, J. D. Park, H. M. Park, N. W. Song, B. S. Shin, D. Marshak, K. Ahn, J. E. Lee, and I. J. Yu, Part. Fibre Toxicol. 10, 36 (2013).

C. Li, S. Taneda, K. Taya, G. Watanabe, X. Li, Y. Fujitani, Y. Ito, T. Nakajima, and A. K. Suzuki, Inhal. Toxicol. 21, 803 (2009).

M. Martini, P. Perriat, M. Montagna, R. Pansu, C. Julien, O. Tillement, and S. Roux, J. Phys. Chem. C 113, 17669 (2009).

Y. Morishita, Y. Yoshioka, H. Satoh, N. Nojiri, K. Nagano, Y. Abe, H. Kamada, S. Tsunoda, H. Nabeshi, T. Yoshikawa, and Y. Tsutsumi, Biochem. Biophys. Res. Commun. 420, 297 (2012).

G. Oberdörster, J. Ferin, and B. E. Lehnert, Environ. Health Perspect. 102 Suppl 5, 173 (1994).

M. O. Oyewumi, A. Kumar, and Z. Cui, Expert Rev. Vaccines 9, 1095 (2010).

E.-J. Park, E. Bae, J. Yi, Y. Kim, K. Choi, S. H. Lee, J. Yoon, B. C. Lee, and K. Park, Environ. Toxicol. Pharmacol. 30, 162 (2010).

L. J. Peek, C. R. Middaugh, and C. Berkland, Adv. Drug Deliv. Rev. 60, 915 (2008).

J. R. Peralta-Videa, L. Zhao, M. L. Lopez-Moreno, G. de la Rosa, J. Hong, and J. L. GardeaTorresdey, J. Hazard. Mater. 186, 1 (2011). 
D. H. Ramdhan, Y. Ito, Y. Yanagiba, N. Yamagishi, Y. Hayashi, C. Li, S. Taneda, A. K. Suzuki, G. Watanabe, K. Taya, M. Kamijima, and T. Nakajima, Toxicol. Lett. 191, 103 (2009).

L. Reimer, Transmission Electron Microscopy, Second Edition (Springer-Verlag, 1989).

M. Rolland, J. Le Moal, V. Wagner, D. Royère, and J. De Mouzon, Hum. Reprod. Oxf. Engl. 28, 462 (2013).

R. Tassinari, F. Cubadda, G. Moracci, F. Aureli, M. D’Amato, M. Valeri, B. De Berardis, A. Raggi, A. Mantovani, D. Passeri, M. Rossi, and F. Maranghi, Nanotoxicology 8, 654 (2014).

D. B. Williams, Practical Analytical Electron Microscopy in Materials Science (Philips Electronic Instruments, 1984).

Y. Xu, N. Wang, Y. Yu, Y. Li, Y.-B. Li, Y.-B. Yu, X.-Q. Zhou, and Z.-W. Sun, PloS One 9, e101572 (2014).

S. Yoshida, K. Hiyoshi, T. Ichinose, H. Takano, S. Oshio, I. Sugawara, K. Takeda, and T. Shibamoto, Int. J. Androl. 32, 337 (2009).

M. van der Zande, R. J. Vandebriel, E. Van Doren, E. Kramer, Z. Herrera Rivera, C. S. Serrano-Rojero, E. R. Gremmer, J. Mast, R. J. B. Peters, P. C. H. Hollman, P. J. M. Hendriksen, H. J. P. Marvin, A. A. C. M. Peijnenburg, and H. Bouwmeester, ACS Nano 6, 7427 (2012). 


\section{FIGURES}
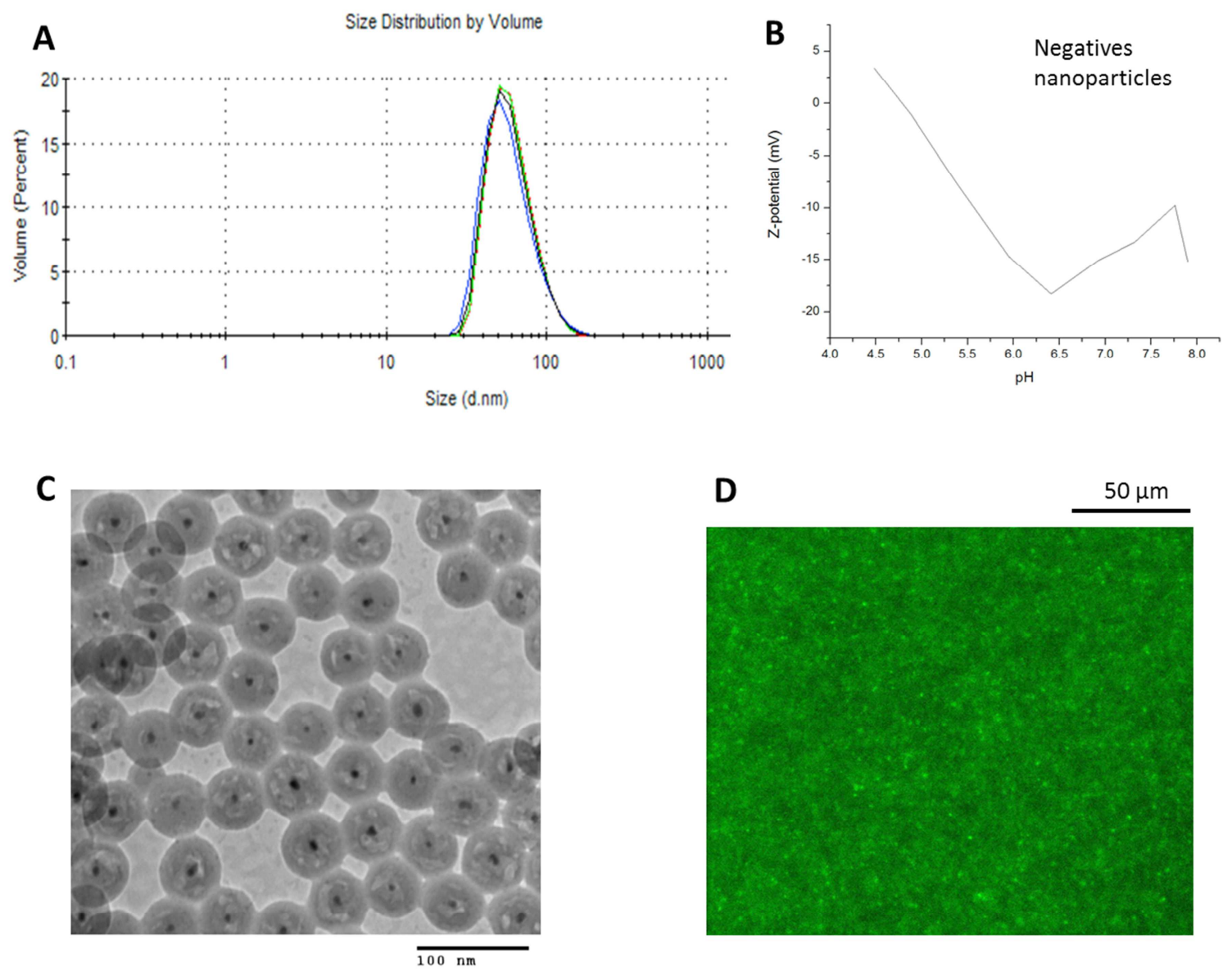

Figure 1: Physico-chemical characteristics of the nanoparticles injected into mice. A) Size distribution obtained by dynamic light scattering. B) Zeta potential (isoelectric $\mathrm{pH}$ ). C) Transmission electron microscopy. Note the reproducibility of the 5-nm gold core. D) FITC fluorescence of the nanoparticles detected by epi-fluorescence microscopy. 
A

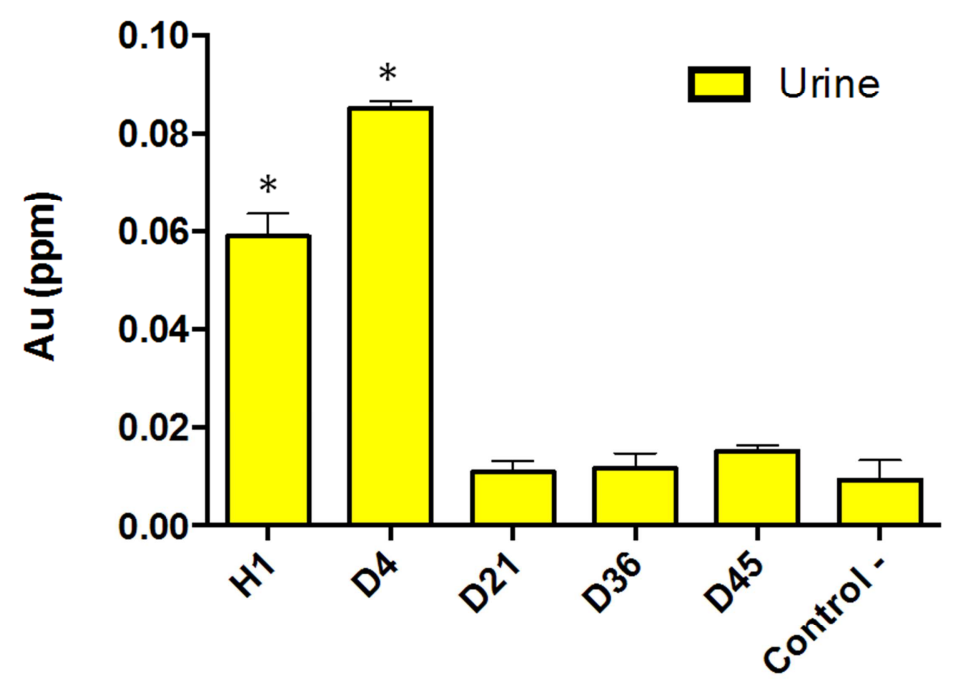

B

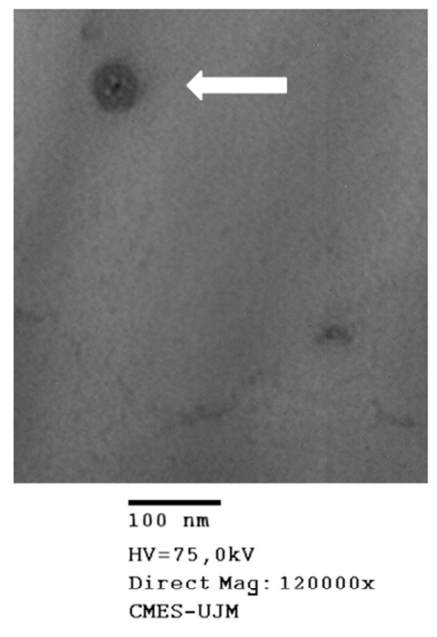

Figure 2: Assessment of nanoparticles detection, morphological stability and ability to reach blood circulation after injection. (A) Quantification of gold in urine by means of ICP-AES. Results are expressed in parts per million (ppm). (B) Representative images of blood samples observed by TEM after filtration. White arrow shows a nanoparticle at D21. 


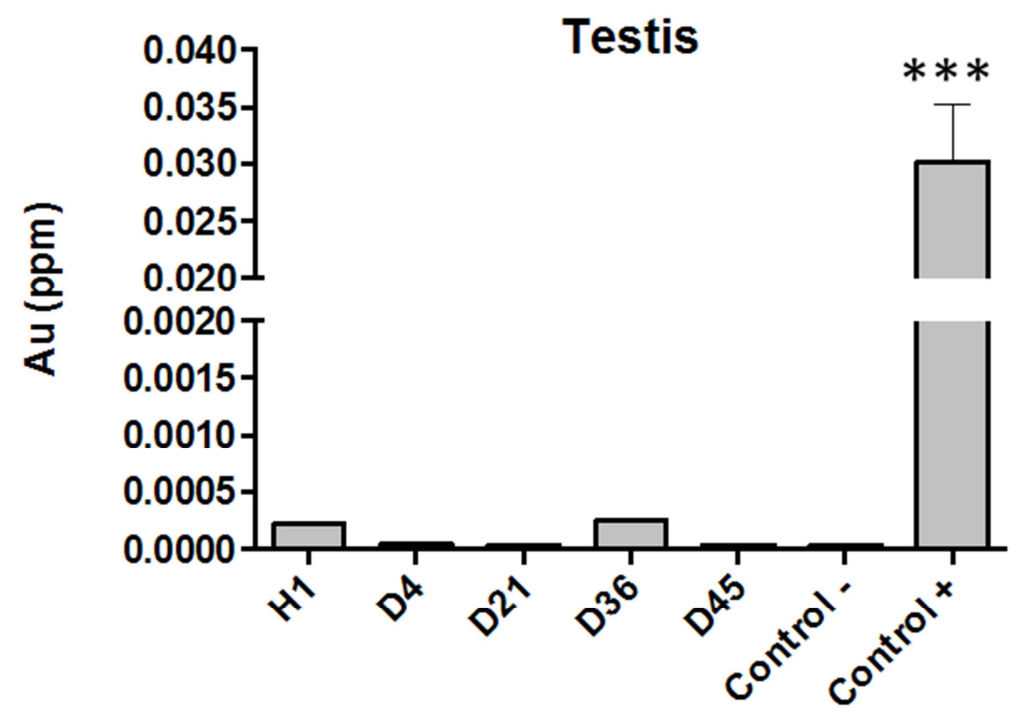

Figure 3: ICP quantification of gold in the testes. Results are expressed in parts per million (ppm). Values are extremely low in each case and the data are not significantly different from the negative control (one-way ANOVA with Bonferroni comparison test, ***p $<0.001$, Graphpad Prism 6). 

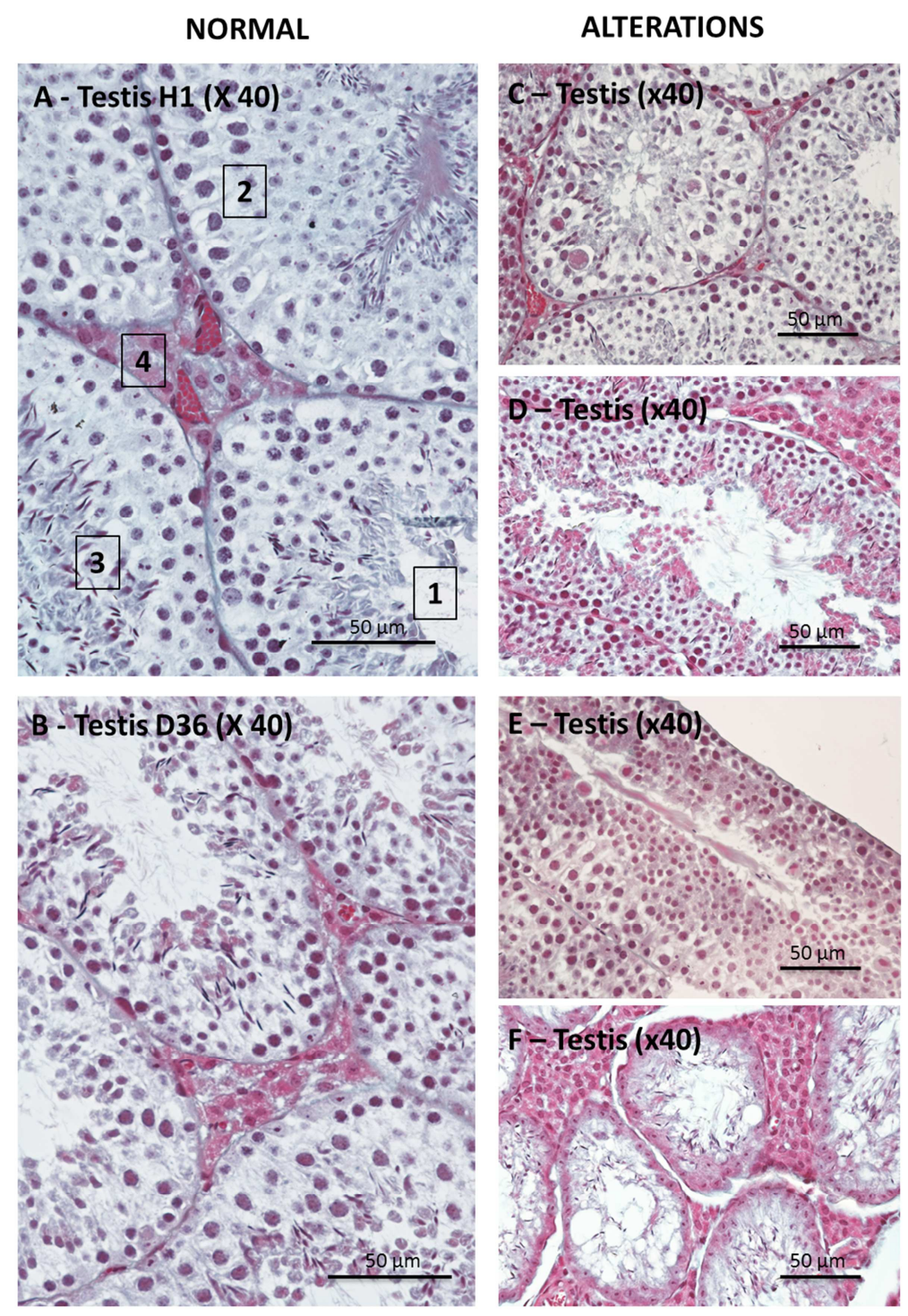

Figure 4: Representative images of histological slides of testis. The left part of the figure shows images of H1 (A) and D36 (B) with normal morphological figures (equivalent to the control condition): (1) seminiferous tubule in cross-section (large tubular structure), (2) different types of germinal cells, (3) sperm (black, tiny, ovoid bodies), (4) tubular spaces with blood vessels and Leydig cells. The right part of the figure illustrates the different histological alterations observed. (C) Giant germinal cells with a large vacuolized cytoplasm, (D) desquameous germinal cells in the tubular lumen, $(\mathrm{E})$ both, $(\mathrm{F})$ empty tubule with only Sertoli cells. 

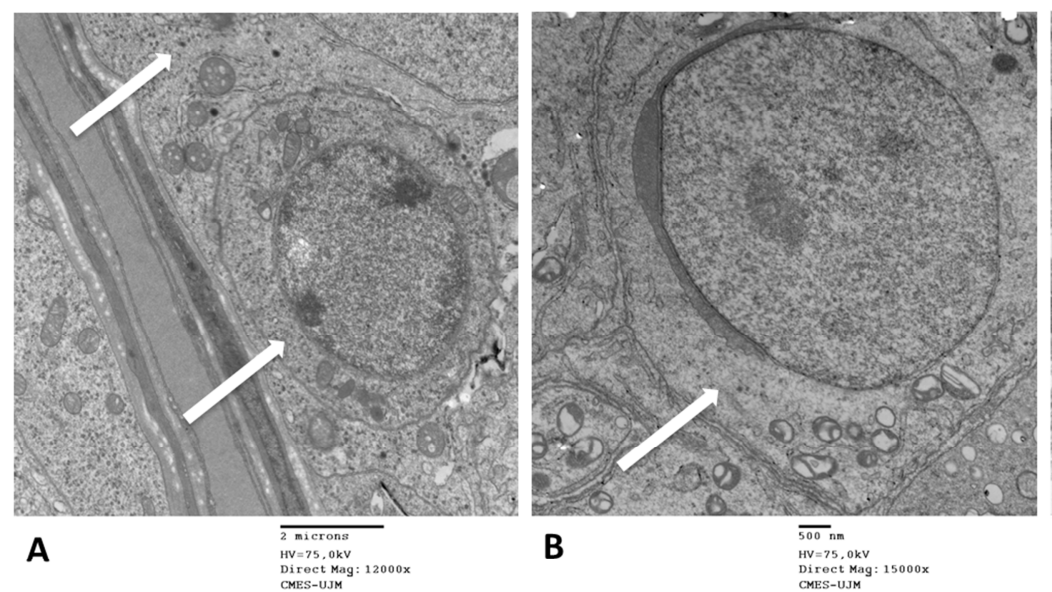

B
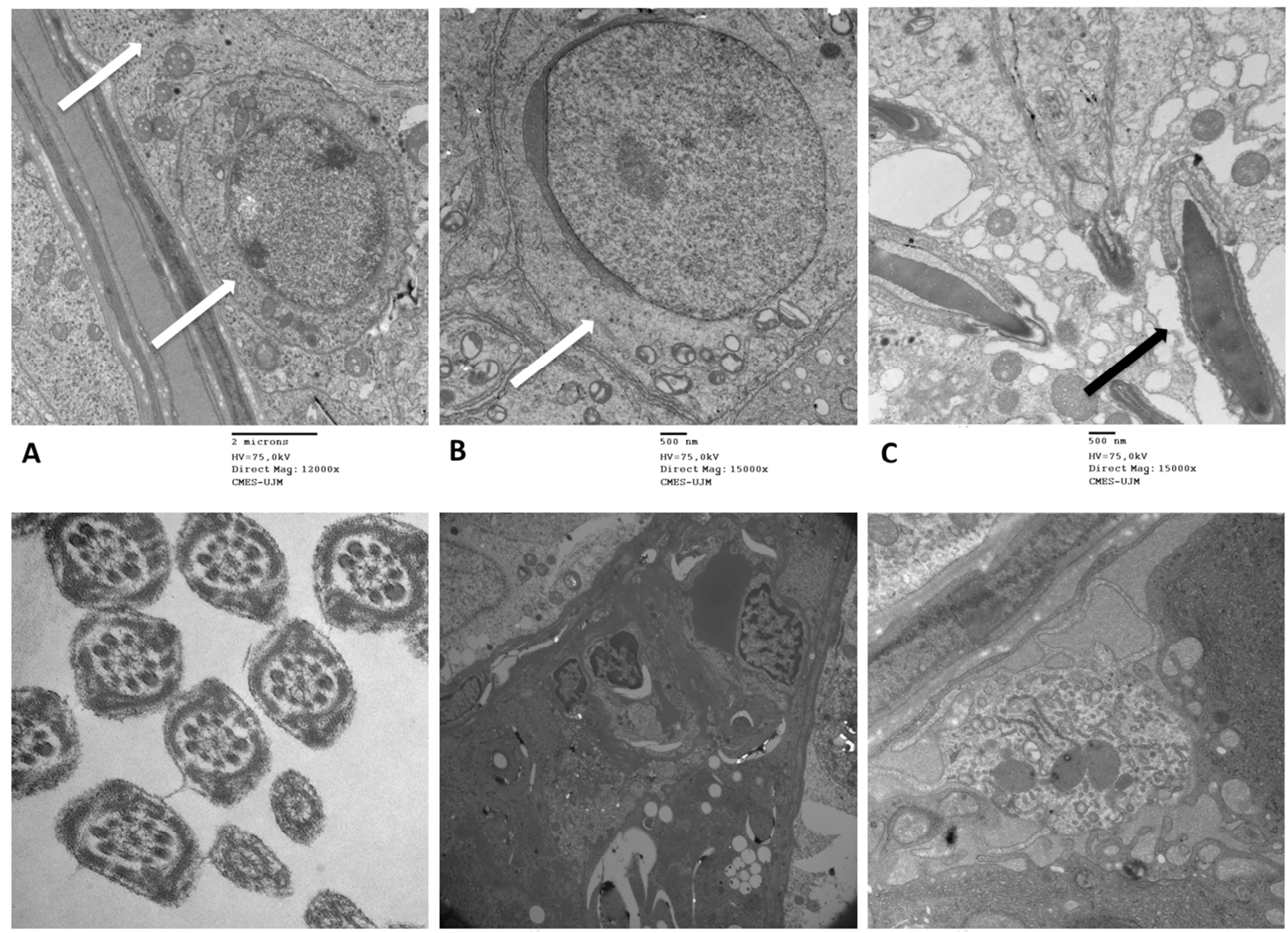

D

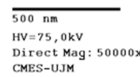

E

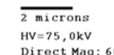

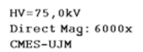

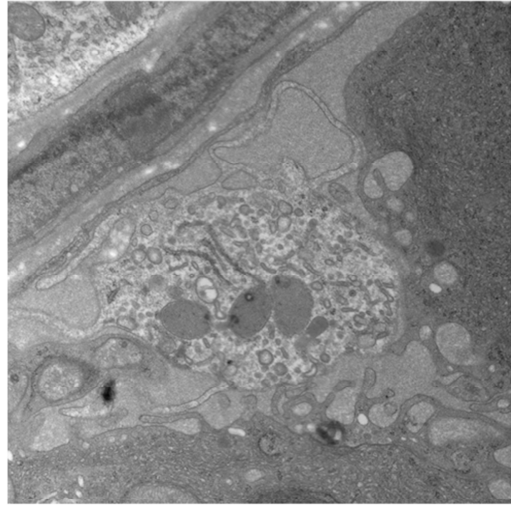

$\mathbf{F}$

$\overline{\substack{500 \mathrm{~nm} \\ \text { HV=75, } 0 \mathrm{~kg},}}$

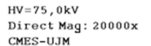

Figure 5: Representative images of testis samples observed by TEM (H1, D4, D21, D36, and D45). (A) Germinal spermatogonia cell contiguous to the basement membrane of the seminiferous tubules (lower white arrow) and Sertoli cell forming the blood-testis barrier (upper white arrow). (B) Spermatocyte (white arrow). (C) Mature spermatozoids (black arrow). (D) Flagellum cross sections. (E, F) Intertubular spaces with Leydig cells, immune cells and vessels. No nanoparticles were detected in the H1, D4, D21, D36. and D45 samples. 

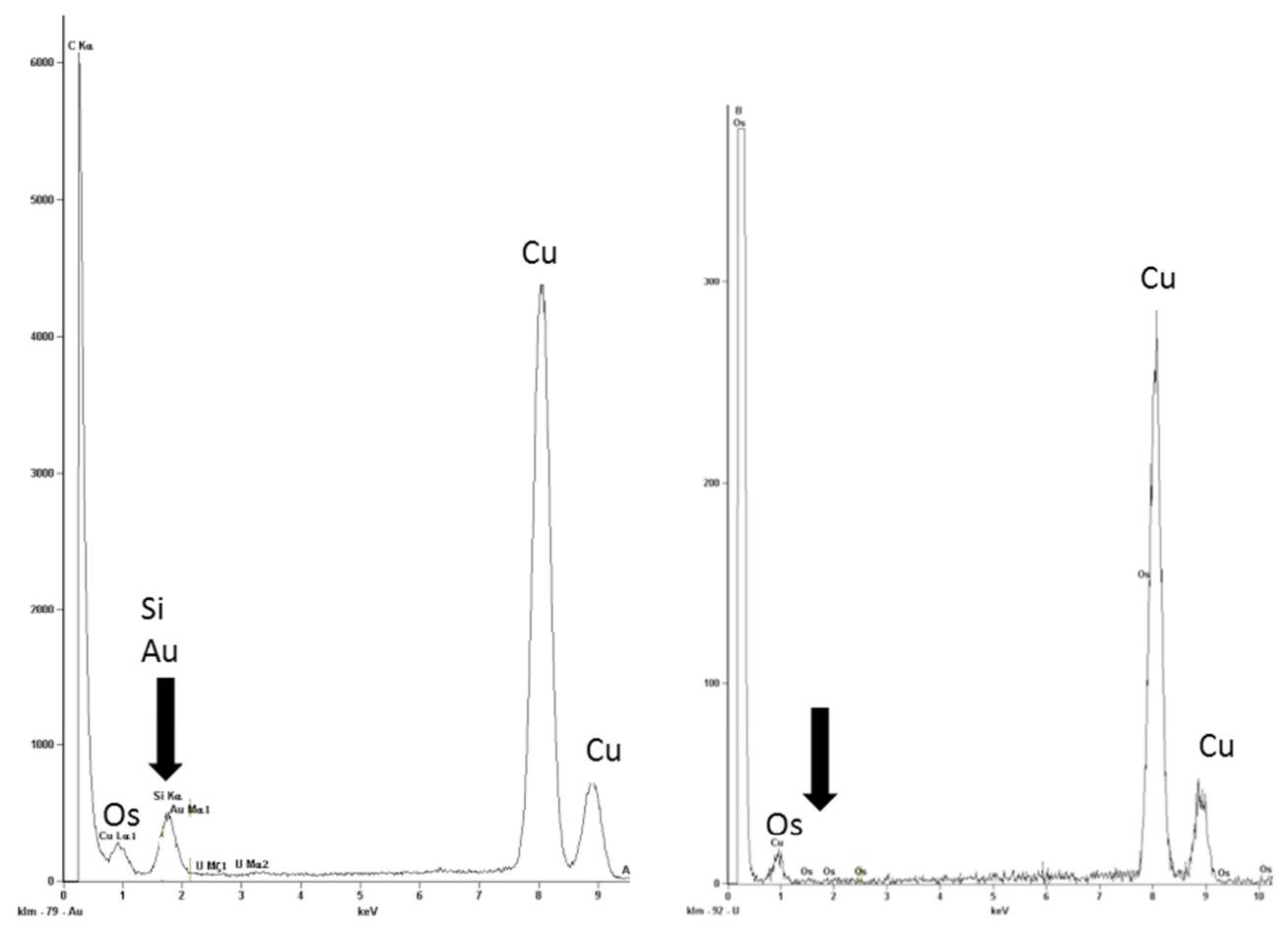

A

B
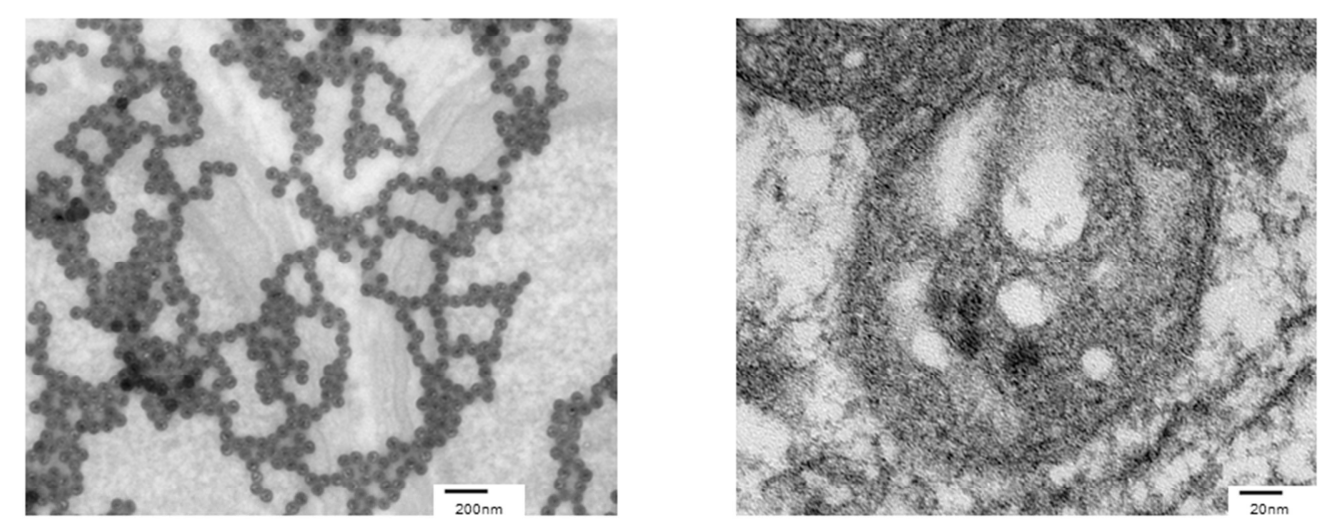

Figure 6: HR imaging studies coupled with EDX analysis in the TOPCON electron microscope for different specimens of interest. The upper part of the figure displays the EDX spectra and the lower part shows the corresponding images of the analyzed regions. A) Control specimen corresponding to a drop of silica-gold nanoparticle suspension deposited on a sample slice. B) Image and spectrum from a D45 sample (representative of all the samples). 

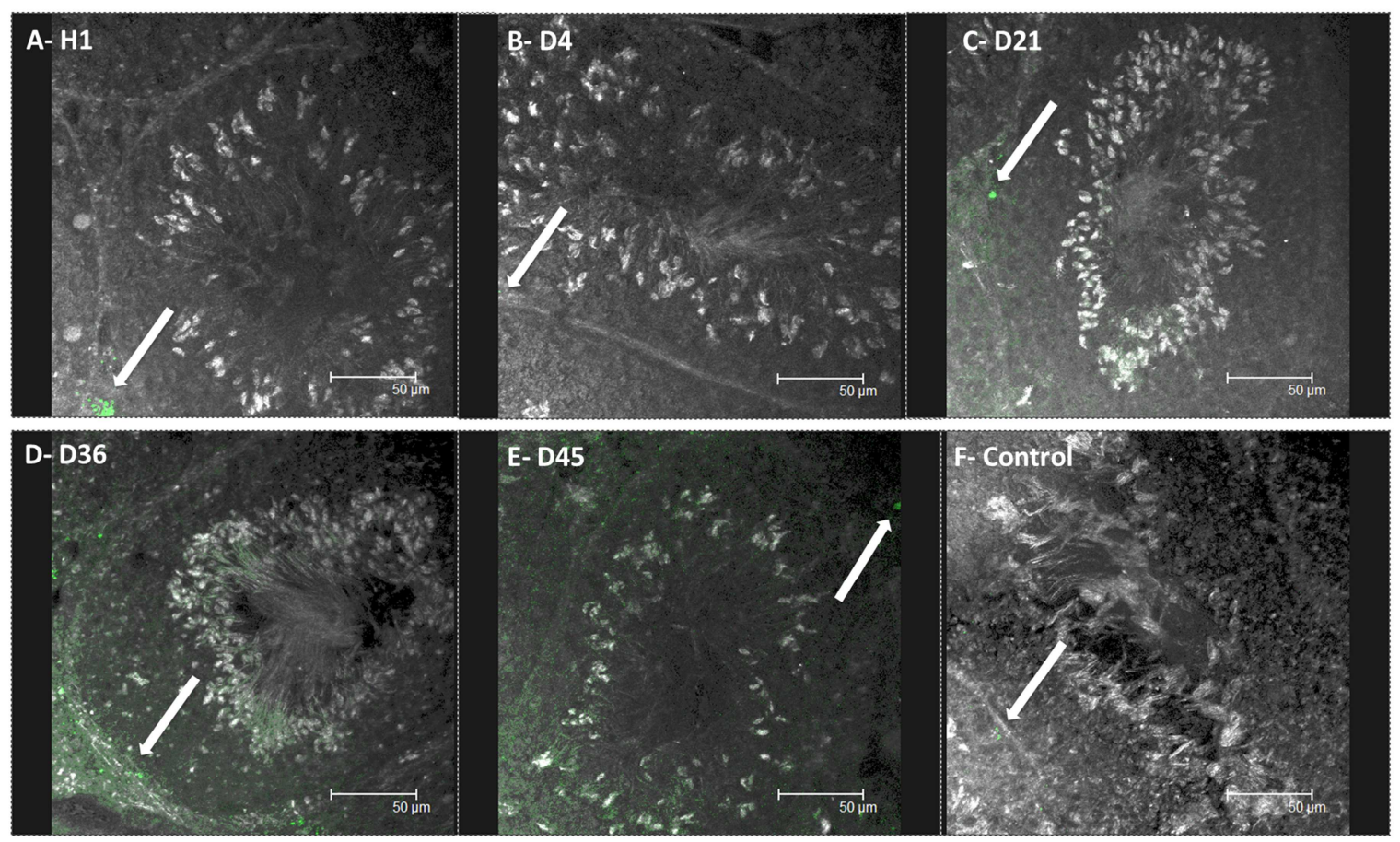

Figure 7: Confocal microscopy observations at the different times of sacrifice (A to $\mathrm{H}$ respectively: H1, D4, D21, D36, D45 and the control). The testes were imaged by reflection (gray level). The green fluorescence observed is not specific of the nanoparticles but instead corresponds to red blood cells (white arrow). 